dieses Buch mitgewidmet ist, und dessen Einfluss sich auch schon im Titel andeutet.

Der Gesamtentwurf des Buches folgt Vorlesungen, die Schmidt an unterschiedlichen Hochschulen gehalten hat, wobei den einzelnen Kapiteln andere Publikationen und Vorträge zugrunde liegen, die für die vorliegende Publikation umfassend überarbeitet, aufeinander abgestimmt und ergänzt wurden. Dennoch gewinnt der aufmerksame Leser des gesamten Buches an der einen oder anderen Stelle den Eindruck, als wäre das Werk nicht ganz aus einem Guss, etwa wenn er über z. T. fast wörtliche Redundanzen stolpert. Offenbar möchte Schmidt dem allgemeinen Trend zur selektiven Lektüre Genüge leisten, indem er jedes Kapitel auf dessen eigenständige Lesbarkeit hin konzipiert hat. Weitere Konzilianz dem Leser (mit begrenzten zeitlichen Ressourcen) gegenüber beweist ein zusammenfassendes Fazit am Ende eines jeden Kapitels sowie ein Rückblick zum Abschluss des Buches. Ein Sachregister erleichtert ferner die Orientierung, wohingegen ein Personenregister leider fehlt. In der Gesamtschau handelt es sich beim „Anderen der Natur" um ein sehr gelungenes Buch, das einen umfassenden und dennoch scharfen Blick auf Natur-, Wissenschafts- und Technikphilosophie wirft.

$\langle\gg$

\section{Die Risiken der Energiewende - Oder die Gefahren des Nichtstuns}

\section{Gross, R. Mautz: Renewable Energies. London, New York: Routledge 2015, 176 S., ISBN 978-1138194519, Euro 38,00}

\section{Rezension von Christian Büscher, Institut für Technikfolgenabschätzung und Systemanalyse (ITAS), Karlsruhe}

In den letzten Jahren sind einige sozialwissenschaftliche Werke zum Thema Erneuerbare Energie (EE), Energiewende oder Transformation des Energiesystems erschienen. Das vorliegende Buch von Matthias Gross und Rüdiger Mautz unterscheidet sich nicht in dem gemeinhin geteilten bias, dass die Verbreitung erneuerbarer Energie- quellen wünschenswert ist - mehr oder weniger optimistisch vertreten alle SozialwissenschaftlerInnen diese Ansicht -, sondern, dass sie zurecht auf das Nichtwissen hinsichtlich dieses Transformationsexperiments, und die Effekte, die dieses Nichtwissen haben kann, hinweisen. Die Energiewende wird stets als Projekt dargestellt, das, wenn es erstmal abgeschlossen ist, eine bessere Situation für alle Betroffenen bereithält. Nur, wer will das mit Sicherheit wissen?

Die Problemstellung dieses Buches ist, etwas grob formuliert: „Our focus for the remainder of this book is on transition-related challenges and their sociological implications for twenty-first-century societies, particularly with regard to the switch from fossil and nuclear to energy drawn from the sun, gravitation, or the Earth's heat" (S. 10). Erst im Laufe der Lektüre wird der Leser erfahren, für wen die Herausforderungen als solche auftreten. Viel dreht sich um die Unsicherheit des Scheiterns der Transition, also die Kontingenz-Erfahrung von Akteuren, die ihre aktive Teilnahme verweigern oder gar opponieren könnten. Bei diesen Akteuren handelt es sich vornehmlich um Personen, Gruppen, Genossenschaften, kleine Organisationen, in denen die Autoren wesentliche Treiber der Wende sehen, und die alle außerhalb des „Energieestablishments" operieren.

Die Autoren stellen keinen eigenen Ansatz vor, sondern lehnen sich durchgängig an Argumente der klassischen Soziologie oder zeitgenössischer Forschungen zur System-Transition (Transition Research) an. Ab und an kommen Argumente aus den eigenen Forschungen zu „Real-Experimenten" zum Vorschein, die mit der Konstruktion aus der Multi-Level-Perspective (z. B. Geels 2002), der „Nische“, verglichen und auf ihre strukturelle Äquivalenz hin untersucht werden (S. 41f.).

\section{Soziologische Frühwarnung}

Es ist interessant zu lesen, wie schon die soziologischen Altforderen (Weber, Simmel) vor den Konsequenzen der fossilen, industriezeitlichen Energiewende (vornehmlich: Kohle) gewarnt haben. Nutzbare Energie war stets ein Treiber gesellschaftlicher Entwicklungen, speziell der industriell-kapitalistischen Dynamik. Kapita- 
listische Wirtschaftsformen sowie Lebens- und Arbeitsstile, die zur fortwährenden Suche nach Energieträgern antreiben, heizen einen sich selbst verstärkenden Prozess an: Technische und organisatorische Mittel haben es ermöglicht, einen Überschuss an Energie zu erschließen, was wiederum neue technische und organisatorische Produktionsweisen ermöglicht habe (S. 31). Simmel und Weber, hier als Zeitzeugen der Industrialisierung herangezogen, warnen vor dem nicht notwendigen, allein am Möglichen angepassten Energieverbrauch, und legen die sozialstrukturelle Bedingungen für die Produktion von Überfluss dar (S. 22f.). Die berühmte Protestantische Ethik (Weber) wird von Gross und Mautz als Doppelmoral entlarvt, weil sie einerseits die göttliche Schöpfung ehre und zum nachhaltigen, moderaten Umgang mit der Natur mahne, nur um gleichzeitig die Forcierung rationaler Arbeitsweisen und des Energieverbrauchs zu feiern. Schlussendlich führen die Autoren Simmel an als Warner vor der Resilienz dominanter Energieregime, die alternative Energieerzeugungsformen in ihrer Entwicklung zu behindern scheinen: „Scientifically informed energy cartels that may generally tend to slow down the processes needed to imagine and devise novel forms of energy utilization“" (S. 23).

\section{Verlernen und Lernen}

Wie können sich nun neue Formen der Bereitstellung und Nutzung von Energie durchsetzen? Zunächst einmal gehe kein Weg vorbei an dem willentlichen Verlernen von technischen Möglichkeiten (und sozialen Routinen bzw. Gewohnheiten). Nur durch Prozesse der „exnovation“, d. h. „discarting technologies“ (S. 3), ließen sich neue Freiheitsgrade für alternative Energieformen finden und implementieren. Das hört sich erstmal trivial an: Man lässt das Alte, um etwas Neues zu probieren. Aber was bedeutet es, ein neues „Energiesystem“ zu probieren? Der Schlüssel dazu sind nach Gross und Mautz Experimente. Mit deren Hilfe lassen sich Lernerfahrungen provozieren, die durch Überraschungen generiert werden. In diesen kontrollierten, selektiven Settings (Kausalitäten werden versucht $\mathrm{zu}$ isolieren) ist die Widerlegung von
Annahmen willkommen, gerade weil diese auf Nicht-Wissen hinweisen. Wie dem auch sei, bei System-Transformationen hingegen sind Fehler gerade nicht willkommen, weil die Leistungserstellung permanent erwartet wird, und weil Fehler zu Konsequenzen nicht nur für die Experimentierenden, sondern auch für die sog. „Beistehenden" - die als potenziell Betroffene abwesenden Anwesenden - führen könnten. Grundsätzlich seien Realexperimente aber notwendig, weil im Rahmen von System-Transformationen in isolierten Verhältnissen erprobte Technologien ihr Funktionieren auch auf der Ebene des Gesamtsystems erweisen müssen (S. 62). Die „Energiewende“ ist nun das Paradebeispiel für ein solches Realexperiment mit hoher inklusiver Wirkung, argumentieren die Autoren: „Research on energy has increasingly turned society into a laboratory - one in which the energy user and non-scientist can potentially play an active part in the experiment, be it via civil society engagement, novel forms of public participation, energy cooperatives, or social networks“" (S. 140).

\section{Governance der Energiewende}

Einen besonderen Stellenwert sprechen die Autoren dem schillernden Begriff der "Governance“ zu. Es sei zwar Government (Politik) gewesen, die Nischenentwicklungen befördert habe, um Real-Experimente anzustoßen; nichtsdestotrotz habe sich daraus eine Dynamik ergeben, die über Regierungs- und Planungsziele hinaus gehend, die Energie-Infrastruktur durch ein Zusammenspiel aus dezentralen technischen Systemen (Windräder, PV-Anlagen, Biogas-Anlagen etc.) und einer Vielzahl neuer Akteuren in ein emergentes Neues haben evolvieren lassen. Dabei sei die traditionelle, korporatistische Verknüpfung von Großindustrie und Staat, so das Argument von Gross und Mautz, unter Druck geraten (ironischerweise auch wenn diese enge Verknüpfung nicht ganz freiwillig aufrecht erhalten bleibt, wie im Fall der grün-roten Landesregierung in Baden-Württemberg und dem EnBW-Konzern). Dazu geselle sich ,growing societal consensus about the need to tackle environmental problems", stellen die Autoren fest, wobei Meinen und Handeln ja oft auseinanderfallen (der Fach- 
begriff der kognitiven Dissonanz hat es schon in die Foren von Tageszeitungen geschafft ${ }^{1}$ ).

Im Weiteren wird dem Leser die Sympathie der Autoren für bestimmte Formen der Governance vorgeführt. In einer Mischform aus Analyse und Wunschdenken werden genossenschaftlich operierende Akteure als Träger der Energiewende stilisiert. Diese zögen ihre Motivation aus einem "diverse set of activities that go far beyond power generation to also include initiatives in energy efficiency, energy consulting, local or regional energy independence, boosting the local economy, improving social cohesion, and so on" (S. 99). Man wird beobachten müssen, inwieweit dieses Modell der dezentralen Diffusion von EE-Innovationen trägt, auch und gerade wenn große Unternehmen in das Geschäft mit EE (wieder-)einsteigen und ihre Einflusssphären ausdehnen. Auch die bereits als Dinosaurier verunglimpften "Großen Vier" (Energiekonzerne) richten ihre Strategie auf EE-Technologien aus (und versuchen, alte Technologie auszulagern).

Es ist ein großer Verdienst dieses Buches, auf die Effekte des Realexperiments „Energiewende" hinzuweisen. Die Betroffenen dieses Jahrhundertprojekts drohen sich möglicherweise enttäuscht abzuwenden, wenn negative Auswirkungen wahrgenommen werden, sprich Umweltprobleme, Arten- und Landschaftsschutz, Entwertung von Eigentum, das Scheitern von Klein-Investitionen, Preissteigerungen, Datenschutzprobleme im sog. „Smart-Grid" etc. Gross und Mautz weisen darauf hin, dass Realexperimente nicht mit den Mitteln des klassischen risk assessment bewertet werden können, weil es an Modellen, die diese gesteigerte Komplexität einfangen könnten, und damit auch an validen Daten mangelt. Deshalb sei auch ein hohes Maß an Nichtwissen involviert. Nun komme es darauf an, ob die Betroffenen den Experten zutrauen, das Nichtwissen zu spezifizieren und durch weitere Forschung in Wissen zu wandeln, oder ob sie nicht spezifizierbares Nichtwissen unterstellen und auf Prinzipiendissens umstellen. Dann wäre das gesamte Projekt womöglich gefährdet: „In this sense, there are good reasons for opposing experimental processes in the transition towards renewable energy" (S. 144). Hier gerät die Dimension des Vertrauens in Experten und Politik in das Schlaglicht.

\section{Ist Erneuerbare Energie ,alternativ“?}

Mittlerweile drohen einige der Argumente, die in diesem Werk vertreten werden, schon wieder von den Entwicklungen überholt zu werden. Erneuerbare Energien werden bereits heute mehr oder weniger offen als die neue „Leittechnologie“ bezeichnet; es bedarf nicht mehr der Nischen, die sie vor dominanten Akteurskonstellationen und Marktkräften schützen. Das macht sich an den politischen Plänen bemerkbar, die Subventionierungen auslaufen zu lassen. Auch muss man sich fragen, ob nicht Netzwerke von Proponenten von EE zukünftige Regime konstituieren, die ihrerseits Weiterentwicklungen blockieren $-\mathrm{z}$. B. wird der bayerischen Landespolitik unterstellt, die idiosynkratischen Investitionen in Biogasanlagen zu schützen. Zudem ist zu vermuten, dass auch Genossenschaften Lobbyismuskräfte entfalten. Insgesamt reduzieren die Autoren die Energiewende auf eine Entscheidung, und welche Neigung sie vertreten ist überdeutlich: Besser handeln als abwarten - auch wenn man noch nicht wissen kann, wie es ausgeht (S. 144).

\section{Anmerkung}

1) Vgl. das Essay von George Monbiot: „Consume more, conserve more: sorry, but we just can't do both" und die vehementen Diskussionen unter den Leserinnen und Lesern (http://www.theguardian. com/commentisfree/2015/nov/24/consume-conserveeconomic-growth-sustainability; download 26.11.15).

\section{Literatur}

Geels, F.W., 2002: Technological Transitions as Evolutionary Reconfiguration Processes: A Multi-level Perspective and a Case-study. In: Research Policy 31 (2002), S. 1257-1274 